\title{
Measure of submanifolds in the Engel group
}

\section{Enrico Le Donne and Valentino Magnani}

\begin{abstract}
We find all intrinsic measures of $C^{1,1}$ smooth submanifolds in the Engel group, showing that they are equivalent to the corresponding $d$-dimensional spherical Hausdorff measure restricted to the submanifold. The integer $d$ is the degree of the submanifold. These results follow from a different approach to negligibility, based on a blow-up technique.
\end{abstract}

\section{Introduction}

Computing the Hausdorff measure of submanifolds in stratified groups with respect to the Carnot-Carathéodory distance is a rather natural question. This may be considered as a first step to study several problems of Geometric Measure Theory in stratified groups.

Nevertheless, this question has not yet an answer. In [3, 0.6 B], Gromov has given a general formula for the Hausdorff dimension of smooth submanifolds in equiregular Carnot-Carathéodory spaces and in [7] it is shown that this formula coincides with the degree of the submanifold, recently introduced in [5]. In the latter work, the authors find an integral formula for the spherical Hausdorff measure of submanifolds in stratified groups under a suitable "negligibility condition". If $d$ is the degree of a submanifold, and $\mathcal{S}^{d}$ is the spherical Hausdorff measure constructed with the Carnot-Carathéodory distance, this condition requires that all points of the submanifold having pointwise degree less than $d$ must be $\mathcal{S}^{d}$-negligible. This negligibility condition has been recently obtained in all two step groups, [7], but it is still open in higher step groups. We address the reader to [5], [6] and [7] for more information on this problem and its connections with the present literature.

2000 Mathematics Subject Classification: Primary 58C35; Secondary 22E25, 53C17. Keywords: Engel group, submanifolds, Hausdorff measure. 
In this work, we prove the negligibility condition in the Engel group, adopting a different approach with respect to the standard covering arguments. Broadly speaking, we simply "blow-up" the points of the submanifold using the intrinsic dilations of the group and then apply a simple fact of Geometric Measure Theory, see Lemma 4. Essentially, we prove that the assumptions of this lemma hold in all the single cases that can occur. In fact, joining all propositions of Section 4 and 5, we have our main result.

Theorem 1. Let $\Sigma$ be a p-dimensional $C^{1,1}$ submanifold of degree $d$ in the Engel group, where $p=1,2$. Then for every $x \in \Sigma$ with $d_{\Sigma}(x)<d$, there holds

$$
\lim _{r \rightarrow 0} \frac{\mu_{p}\left(\Sigma \cap D_{x, r}\right)}{r^{d}}=+\infty
$$

We have denoted by $\mu_{p}$ the $p$-dimensional Riemannian surface measure induced on $\Sigma$ by a fixed left invariant Riemannian metric on the group. The main feature of the previous theorem is that (1.1) not only depends on the degree at $x$, but also on the "behaviour" of $\Sigma$ around $x$, that is expressed by the degree of $\Sigma$. It is rather interesting to observe how the limit (1.1) in some cases requires the use of this "global" information. This is the case of Proposition 9, where the fact that $\Sigma$ has degree three implies a differential constraint, given by the system of PDEs (4.9), that play a crucial role in the proof of (1.1).

Theorem 1 joined with Lemma 4 yields Theorems 5 and 10, that correspond to the negligibility condition stated in (1.5) of [5]. This condition gives (1.4) of [5], namely, we have the following

Corollary 2. Let $\Sigma$ be a p-dimensional $C^{1,1}$ submanifold of degree $d$ in the Engel group. Then the following formula holds

$$
\int_{\Sigma} \theta\left(\tau_{\Sigma}^{d}(x)\right) d \mathcal{S}^{d}(x)=\int_{\Sigma}\left|\tau_{\Sigma}^{d}(x)\right| d \tilde{\mu}_{p}(x)
$$

The $p$-tangent vector $\tau_{\Sigma}^{d}(x)$ is the component of degree $d$ of the $p$-tangent vector $\tau_{\Sigma}(x)$ associated to the tangent space $T_{x} \Sigma$. Its norm is computed with respect to the auxiliary Riemannian metric fixed on the group. This metric also yields the surface measure $\tilde{\mu}_{p}$ induced on $\Sigma$, see [5] for more details. In the case $p=3$, the previous integral formula follows from Theorem 2.20 of [4]. In fact, Frobenius Theorem implies that $C^{1,1}$ hypersurfaces in any stratified group must possess non-horizontal points, hence they have degree equal to $Q-1$, where $Q$ is the Hausdorff dimension of the group. Recall that non-horizontal points have been introduced in [5] and studied in [6]. According to Proposition 3.2 of [7], the length of the horizontal normal $\left|n_{H}(x)\right|$ 
in Theorem 2.20 of [4] is equal to the length $\left|\tau_{\Sigma}^{d}(x)\right|$. Of course, 4-dimensional submanifolds of the Engel group are just open subsets, for which it is trivial to observe that their degree is exactly 7 and their 7 -dimensional Hausdorff measure is clearly positive and finite on the intersection with bounded sets. Since the metric factor $\theta\left(\tau_{\Sigma}^{d}(x)\right)$ is uniformly bounded from above and from below, $\mathcal{S}^{d}\left\llcorner\Sigma\right.$ is equivalent to the intrinsic measure $\left|\tau_{\Sigma}^{d}(x)\right| \tilde{\mu}_{p}\llcorner\Sigma$, introduced in [5]. In case it is possible to find a distance that yields a constant metric factor, then up to a geometric constant, we obtain

$$
\mathcal{S}_{\mathbb{G}}^{d}(\Sigma)=\int_{\Sigma}\left|\tau_{\Sigma}^{d}(x)\right| d \tilde{\mu}_{p}(x)
$$

As an immediate consequence, the degree of a $C^{1,1}$ submanifold in the Engel group equals its Hausdorff dimension, since points of maximum degree form an open subset of the submanifold. In Remark 1 we point out how our results are also related to Gromov's dimension comparison problem recently raised in [1].

\section{Basic definitions and standard results}

The Engel group $\mathbb{E}$ is a connected, simply connected stratified group, whose Lie algebra satisfies the direct decomposition

$$
\mathcal{E}=V_{1} \oplus V_{2} \oplus V_{3}
$$

and there exists a basis $\left(X_{1}, X_{2}, X_{3}, X_{4}\right)$ of $\mathcal{E}$, such that the only nontrivial brackets are

$$
\left[X_{1}, X_{2}\right]=X_{3} \text { and }\left[X_{1}, X_{3}\right]=X_{4},
$$

where $V_{1}=\operatorname{span}\left\{X_{1}, X_{2}\right\}, V_{2}=\operatorname{span}\left\{X_{3}\right\}$ and $V_{3}=\operatorname{span}\left\{X_{4}\right\}$. We represent the Engel group $\mathbb{E}$ by $\mathbb{R}^{4}$ equipped with the vector fields

$$
X_{1}=\partial_{1}, \quad X_{2}=\partial_{2}+x_{1} \partial_{3}+\frac{x_{1}^{2}}{2} \partial_{4}, \quad X_{3}=\partial_{3}+x_{1} \partial_{4}, \quad X_{4}=\partial_{4},
$$

where the associated exponential mapping builds the group operation in $\mathbb{R}^{4}$ that makes it isomorphic to the abstract Engel group. The intrinsic dilations $\delta_{r}: \mathbb{R}^{4} \longrightarrow \mathbb{R}^{4}$ are given by $\delta_{r}(x)=\left(r x_{1}, r x_{2}, r^{2} x_{3}, r^{3} x_{4}\right)$, with $r>0$. This is a one parameter family of group automorphisms, since

$$
\left(\delta_{r}\right)_{*}\left(X_{j}\right)= \begin{cases}r X_{j} & \text { if } j=1,2 \\ r^{2} X_{3} & \text { if } j=3 \\ r^{3} X_{4} & \text { if } j=4\end{cases}
$$

as one can check from direct computation. 
We fix a left invariant Riemannian metric in $\mathbb{R}^{4}$ that makes $X_{j}$ 's orthonormal. The Carnot-Carathéodory distance associated to $\operatorname{span}\left\{X_{1}, X_{2}\right\}$ along with the fixed left invariant metric on $\mathbb{R}^{4}$ yields a homogeneous distance. More generally, we will consider an arbitrary homogeneous distance $\rho$ on $\mathbb{R}^{4}$, namely, a continuous, left invariant distance that satisfies

$$
\rho\left(\delta_{r} x, \delta_{r} y\right)=r \rho(x, y) \quad \text { for every } \quad x, y \in \mathbb{R}^{4}, \quad r>0 .
$$

In the sequel, the abstract Engel group $\mathbb{E}$ will be identified with $\mathbb{R}^{4}$, equipped with left invariant vector fields (2.1), distance $\rho$ and dilations $\delta_{r}$. The explicit formula for the group operation in $\mathbb{R}^{4}$ will not be needed.

Our arguments are based on the following elementary fact of Geometric Measure Theory, see for instance [2, 2.10.19].

Lemma 3. Let $X$ be a metric space, let $\mu$ be a Borel measure on $X$ and let $\left\{V_{i}\right\}_{i \in \mathbb{N}}$ be an open covering of $X$ such that $\mu\left(V_{i}\right)<\infty$. Let $Z \subset X$ be a Borel set and suppose that

$$
\limsup _{r \rightarrow 0^{+}} r^{-a} \mu\left(D_{x, r}\right) \geq \kappa>0
$$

whenever $x \in Z$, where $a>0$. Then $\mu(Z) \geq \kappa \mathcal{S}^{a}(Z)$.

We have denoted by $\mathcal{S}^{a}$ the $a$-dimensional spherical Hausdorff measure constructed with the size function $\zeta_{a}\left(D_{x, r}\right)=r^{a}$ and $D_{x, r}$ is the closed ball of center $x$ and radius $r$. From the previous lemma, we get the straightforward

Lemma 4. Let $\Sigma$ be $k$-dimensional $C^{1,1}$ submanifold of $\mathbb{E}$ and let $\mu_{k}$ be the left invariant Riemannian measure of $\mathbb{E}$ restricted to $\Sigma$. If $Z$ is a Borel set of $\Sigma$ such that $\limsup _{r \rightarrow 0^{+}} r^{-a} \mu_{k}\left(D_{z, r}\right)=+\infty$, whenever $z \in Z$, then $\mathcal{S}^{a}(Z)=0$.

\section{Degree of submanifolds in the Engel group}

The degree of a 2-vector $\tau=\sum_{1 \leq i<j \leq 4} \tau_{i j} X_{i} \wedge X_{j} \in \Lambda_{2}(\mathcal{E})$ is given by

$$
\operatorname{deg}(\tau)=\max \left\{d_{i}+d_{j} \mid \tau_{i j} \neq 0\right\}
$$

where $d_{i}$ is the degree of $X_{i}$, hence $d_{1}=d_{2}=1, d_{3}=2$ and $d_{4}=3$. Analogously, the degree of a vector $\tau=\sum_{i=1}^{4} \tau_{i} X_{i} \in \mathcal{E}$ is given by $\operatorname{deg}(\tau)=$ $\max \left\{d_{i} \mid \tau_{i} \neq 0\right\}$. Then we define the pointwise degree at $x$ of a 2 dimensional submanifold $\Sigma$ in $\mathbb{E}$ as

$$
d_{\Sigma}(x)=\operatorname{deg}\left(\tau_{\Sigma}(x)\right),
$$

where $\tau_{\Sigma}(x)$ is the 2-tangent vector of $\Sigma$ at $x \in \Sigma$. The degree $d(\Sigma)$ of $\Sigma$ is the integer $\max _{x \in \Sigma} d_{\Sigma}(x)$, see [5] for more details in the general case of stratified groups. 
Let $U$ be an open subset of $\mathbb{R}^{2}$ and let $\phi: U \longrightarrow \mathbb{R}^{4}$ be a $C^{1}$ immersion. According to computations in Section 4 of [5], we have

$$
\begin{aligned}
\phi_{u_{1}} \wedge \phi_{u_{2}} & =\phi_{u}^{12} X_{1} \wedge X_{2}+\left(\phi_{u}^{13}-\phi^{1} \phi_{u}^{12}\right) X_{1} \wedge X_{3}+\phi_{u}^{23} X_{2} \wedge X_{3} \\
& +\left(\phi_{u}^{14}-\phi^{1} \phi_{u}^{13}+\frac{\left(\phi^{1}\right)^{2}}{2} \phi_{u}^{12}\right) X_{1} \wedge X_{4}+\left(\phi_{u}^{24}-\phi^{1} \phi_{u}^{23}\right) X_{2} \wedge X_{4} \\
& +\left(\phi_{u}^{34}+\frac{\left(\phi^{1}\right)^{2}}{2} \phi_{u}^{23}-\phi^{1} \phi_{u}^{24}\right) X_{3} \wedge X_{4},
\end{aligned}
$$

where we have defined

$$
\phi=\left(\phi^{1}, \phi^{2}, \phi^{3}, \phi^{4}\right) \quad \text { and } \quad \phi_{u}^{i j}=\operatorname{det}\left(\begin{array}{cc}
\phi_{u_{1}}^{i} & \phi_{u_{2}}^{i} \\
\phi_{u_{1}}^{j} & \phi_{u_{2}}^{j}
\end{array}\right) .
$$

It is also understood that $X_{i} \wedge X_{j}$ in the previous formula are evaluated at the point $\phi(u)$. Thus, if $\phi$ locally parametrizes a surface $\Sigma$, according to the notion of pointwise degree, we have that

$$
d_{\Sigma}(\phi(u))= \begin{cases}5 & \text { if } c_{34}(u) \neq 0 \\ 4 & \text { if }\left|c_{14}(u)\right|+\left|c_{24}(u)\right|>0 \text { and } c_{34}(u)=0 \\ 3 & \text { if }\left|c_{13}(u)\right|+\left|c_{23}(u)\right|>0 \text { and } c_{34}(u)=c_{14}(u)=c_{24}(u)=0 \\ 2 & \text { if } c_{34}(u)=c_{14}(u)=c_{24}(u)=c_{13}(u)=c_{23}(u)=0\end{cases}
$$

where we have set

$$
\phi_{u_{1}} \wedge \phi_{u_{2}}=\sum_{1 \leq i<j \leq 4} c_{i j}(u) X_{i} \wedge X_{j} .
$$

Concerning curves, we use again computations in Section 4 of [5]. We consider a $C^{1}$ immersion $\phi: I \longrightarrow \mathbb{R}^{4}$, where $I$ is an open interval of $\mathbb{R}$. Thus, we get

$$
\dot{\phi}=\dot{\phi}^{1} X_{1}+\dot{\phi}^{2} X_{2}+\left(\dot{\phi}^{3}-\phi^{1} \dot{\phi}^{2}\right) X_{3}+\left(\dot{\phi}^{4}-\phi^{1} \dot{\phi}^{3}+\frac{\left(\phi^{1}\right)^{2}}{2} \dot{\phi}^{2}\right) X_{4} .
$$

In analogous way, defining $\dot{\phi}=\sum_{i=1}^{4} c_{i}(t) X_{i}$, we have

$$
d_{\Sigma}(\phi(t))= \begin{cases}3 & \text { if } c_{4}(u) \neq 0 \\ 2 & \text { if } c_{3}(t) \neq 0 \\ 1 & \text { if }\left|c_{1}(t)\right|+\left|c_{2}(t)\right|>0 .\end{cases}
$$

Remark 1. By definition of degree, in the Engel group one easily notices that the possible degrees of $C^{1,1}$ surfaces can only be 3,4 or 5 . Degree two is not possible due to the Frobenius Theorem. Of course, curves can only have degrees 1,2 or 3 and again Frobenius Theorem implies that hypersurfaces can only have degree 6 . Thus, these are all possible Hausdorff dimensions of $C^{1,1}$ smooth submanifolds in the Engel group and formula (1.2) holds for them. This answers Problem 1.1 of [1] in the case where the ambient space is the Engel group, see also Section 8.1 of the same paper. 


\section{Surfaces in the Engel group}

In this section we wish to show the following

Theorem 5. Let $\Sigma$ be a 2-dimensional $C^{1,1}$ smooth submanifold of $\mathbb{E}$. Let d be the degree of $\Sigma$ and let $\Sigma_{d}$ be the open subset of points of degree $d$. Then we have

$$
\mathcal{S}^{d}\left(\Sigma \backslash \Sigma_{d}\right)=0
$$

Recall that the degree of a point in a submanifold is invariant under left translations. In the sequel, we will number the independent variables of parametrized surfaces taking into account the coordinate plane they span in $\mathbb{R}^{4}$. In fact, our surfaces are locally presented also as graphs embedded in $\mathbb{R}^{4}$. These indexes will also help us to keep in mind the degree of the corresponding variables with respect to the Engel dilations of $\mathbb{R}^{4}$.

Lemma 6. Let $\Sigma$ be a 2-dimensional $C^{1}$ smooth submanifold of $\mathbb{E}$ and let $x \in \Sigma$. Then there exist local coordinates $u$ in a neighbourhood $U$ of 0 in $\mathbb{R}^{2}$ such that $x^{-1} \Sigma$ around zero is given by the local parametrization $\phi: U \longrightarrow x^{-1} \Sigma$ with $\phi(0)=0$ and we have

$$
\phi(u)= \begin{cases}\left(\phi^{1}(u), \phi^{2}(u), u_{3}, u_{4}\right) & \text { if } d_{\Sigma}(x)=5, \\ \left(u_{1}, \phi^{2}(u), \phi^{3}(u), u_{4}\right) \text { or }\left(\phi^{1}(u), u_{2}, \phi^{3}(u), u_{4}\right) & \text { if } d_{\Sigma}(x)=4, \\ \left(u_{1}, \phi^{2}(u), u_{3}, \phi^{4}(u)\right) \text { or }\left(\phi^{1}(u), u_{2}, u_{3}, \phi^{4}(u)\right) & \text { if } d_{\Sigma}(x)=3, \\ \left(u_{1}, u_{2}, \phi^{3}(u), \phi^{4}(u)\right) & \text { if } d_{\Sigma}(x)=2,\end{cases}
$$

where the functions $\phi^{j}$ satisfy

$$
\begin{cases}\nabla \phi^{3}(0)=\left(0, \phi_{u_{4}}^{3}(0)\right) & \text { if } d_{\Sigma}(x)=4, \\ \nabla \phi^{4}(0)=(0,0) & \text { if } d_{\Sigma}(x)=3, \\ \nabla \phi^{4}(0)=(0,0) \text { and } \nabla \phi^{3}(0)=(0,0) & \text { if } d_{\Sigma}(x)=2 .\end{cases}
$$

Furthermore, for small $r>0$, we have

$$
\mu_{2}\left(D_{x, r} \cap \Sigma\right)=r^{d_{\Sigma}(x)} \int_{\tilde{\delta}_{1 / r}\left(\phi^{-1}\left(D_{r}\right)\right)} J \phi\left(\tilde{\delta}_{r} u\right) d u
$$

where $J \phi(x)=\sqrt{\operatorname{det}\left(\left\langle\phi_{u_{i}}, \phi_{u_{j}}\right\rangle\right)_{i, j=1, \ldots, 4}}$ is the Riemannian Jacobian of $\phi$ with respect to the fixed left invariant Riemannian metric on $\mathbb{E}$. The induced dilations on coordinates $u$ are defined as $\tilde{\delta}_{r}(u)=\left(r^{d_{i}} u_{i}, r^{d_{j}} u_{j}\right)$, where $d_{1}=$ $d_{2}=1, d_{3}=2$ and $d_{4}=3$. 
Proof. The proof of (4.2) simply follows from the implicit function theorem and (3.1). For example, let us consider the case $d_{\Sigma}(x)=5$ and let $\psi$ any local parametrization of $x^{-1} \Sigma$ around the origin. Then applying (3.1) to $\psi$ at the origin, we must have $\psi_{v}^{34}(0) \neq 0$, since $\psi(0)=0$. Then the mapping

$$
\left(v_{1}, v_{2}\right) \longrightarrow\left(\psi^{3}(v), \psi^{4}(v)\right)
$$

is invertible around the origin and one can take the new coordinates

$$
\left(u_{3}, u_{4}\right)=\left(\psi^{3}(v), \psi^{4}(v)\right) .
$$

The remaining cases proceed in similar way. Now, if we apply (3.1) to the parametrization $\phi$ at the origin, having one of the forms given by (4.2), then a simple computation leads us to (4.3). As an example, let us consider the case $d_{\Sigma}(x)=4$ and assume for instance that $\phi(u)=\left(u_{1}, \phi^{2}(u), \phi^{3}(u), u_{4}\right)$, according to the second formula of (4.2). Then we have

$$
\begin{aligned}
& \operatorname{deg}\left(\left(\phi_{u_{1}} \wedge \phi_{u_{4}}\right)(0)\right)= \\
& \quad=\operatorname{deg}\left(\left(X_{1}+\phi_{u_{1}}^{2}(0) X_{2}+\phi_{u_{1}}^{3}(0) X_{3}\right) \wedge\left(\phi_{u_{4}}^{2}(0) X_{2}+\phi_{u_{4}}^{3}(0) X_{3}+X_{4}\right)\right) \\
& \quad=\operatorname{deg}\left(X_{1} \wedge X_{4}+\phi_{u_{1}}^{2}(0) X_{2} \wedge X_{4}+\phi_{u_{1}}^{3}(0) X_{3} \wedge X_{4}\right) .
\end{aligned}
$$

Taking into account the definition of degree, in the latter equalities we have omitted the linear combinations of $X_{1} \wedge X_{2}, X_{2} \wedge X_{3}$ and $X_{1} \wedge X_{3}$, since they have degree less than four and there is a nonvanishing addend $X_{1} \wedge X_{4}$ of degree four. Observing that $\operatorname{deg}\left(X_{3} \wedge X_{4}\right)=5$, hence we must have $\phi_{u_{1}}^{3}(0)=0$ and the first formula of (4.3) follows. The other cases are achieved in the same way. The left invariance of the Riemannian surface measure gives

$$
\mu_{2}\left(\Sigma \cap D_{x, r}\right)=\mu_{2}\left(x^{-1} \Sigma \cap D_{r}\right)=\int_{\phi^{-1}\left(D_{r}\right)} J \phi(u) d u .
$$

The change of variable $u=\tilde{\delta}_{r}(v)=\left(r^{d_{i}} v_{i}, r^{d_{j}} v_{j}\right)$ and the fact that $d_{\Sigma}(x)=$ $d_{i}+d_{j}$ lead us to formula (4.4).

In the sequel, we will use the following box

$$
\operatorname{Box}_{r}=[-r, r]^{2} \times\left[-r^{2}, r^{2}\right] \times\left[-r^{3}, r^{3}\right]
$$

In fact, setting $D_{r}=D_{0, r}$ and using homogeneity, there exists $\lambda>0$ such that

$$
\operatorname{Box}_{\lambda r} \subset D_{r} \subset \operatorname{Box}_{r / \lambda} \text { for every } r>0 \text {. }
$$

Proposition 7. Let $\Sigma$ be a 2-dimensional $C^{1,1}$ smooth submanifold of $\mathbb{E}$ and assume that $d(\Sigma) \geq 4$ and $d_{\Sigma}(x)=2$. Then we have

$$
\lim _{r \rightarrow 0} \frac{\mu_{2}\left(\Sigma \cap D_{x, r}\right)}{r^{d(\Sigma)}}=+\infty \text {. }
$$


Proof. We use the coordinates given by Lemma 6 and apply formulae (4.2) and (4.3). Then we have a constant $c>0$ such that $\left|\phi^{3}(u)\right| \leq c|u|^{2}$ and $\left|\phi^{4}(u)\right| \leq c|u|^{2}$ for $u$ small. We have

$$
\begin{aligned}
& \tilde{\delta}_{\frac{1}{r}}\left(\phi^{-1}\left(\operatorname{Box}_{\lambda r}\right)\right) \\
& \quad=\tilde{\delta}_{1 / r}\left\{\left(u_{1}, u_{2}\right):\left|u_{1}\right| \leq \lambda r,\left|u_{2}\right| \leq \lambda r,\left|\phi^{3}(u)\right| \leq(\lambda r)^{2},\left|\phi^{4}(u)\right| \leq(\lambda r)^{3}\right\},
\end{aligned}
$$

that can be written as follows

$$
\begin{aligned}
& \tilde{\delta}_{\frac{1}{r}}\left(\phi^{-1}\left(\operatorname{Box}_{\lambda r}\right)\right) \\
& =\left\{\left(x_{1}, x_{2}\right): \frac{\left|x_{1}\right|}{\lambda} \leq 1, \frac{\left|x_{2}\right|}{\lambda} \leq 1, \frac{\left|\phi^{3}\left(r x_{1}, r x_{2}\right)\right|}{(\lambda r)^{2}} \leq 1, \frac{\left|\phi^{4}\left(r x_{1}, r x_{2}\right)\right|}{(\lambda r)^{3}} \leq 1\right\} \\
& \supset\left\{\left(x_{1}, x_{2}\right):\left|x_{1}\right| \leq \lambda,\left|x_{2}\right| \leq \lambda,|x| \leq \frac{\lambda}{\sqrt{c}},|x| \leq \frac{\lambda^{3 / 2}}{\sqrt{c}} \sqrt{r}\right\} .
\end{aligned}
$$

Taking into account (4.4) and (4.5), hence as $r \rightarrow 0$ we get

$$
\frac{\mu_{2}\left(\Sigma \cap D_{x, r}\right)}{r^{d(\Sigma)}} \geq \frac{\pi \lambda^{3}}{c} \frac{J \phi(0)}{2} r^{1-d(\Sigma)+d_{\Sigma}(x)}=\frac{\pi \lambda^{3}}{c} \frac{J \phi(0)}{2} r^{3-d(\Sigma)} \longrightarrow+\infty .
$$

Proposition 8. Let $\Sigma$ be a 2-dimensional $C^{1,1}$ smooth submanifold of $\mathbb{E}$ and assume that $d(\Sigma) \geq 4$ and $d_{\Sigma}(x)=3$. Then we have

$$
\lim _{r \rightarrow 0} \frac{\mu_{2}\left(\Sigma \cap D_{x, r}\right)}{r^{d(\Sigma)}}=+\infty
$$

Proof. Applying (4.2) and (4.3) from Lemma 6 yields a parametrization $\phi(u)$ of a neighbourhood of 0 in $x^{-1} \Sigma$ having one of the following two forms

$$
\phi(u)=\left(u_{1}, \phi^{2}(u), u_{3}, \phi^{4}(u)\right) \quad \text { or } \quad \phi(u)=\left(\phi^{1}(u), u_{2}, u_{3}, \phi^{4}(u)\right)
$$

together with a constant $c>0$ such that either

$$
\left|\phi^{2}(u)\right| \leq c|u| \quad \text { or } \quad\left|\phi^{1}(u)\right| \leq c|u|
$$

and also $\left|\phi^{4}(u)\right| \leq c|u|^{2}$ for $u$ small. Assume for instance that $\phi(u)=$ $\left(u_{1}, \phi^{2}(u), u_{3}, \phi^{4}(u)\right)$. Of course, the proof is the same in the case $\phi$ assumes the other form in $(4.6)$. The set $\tilde{\delta}_{\frac{1}{r}}\left(\phi^{-1}\left(\operatorname{Box}_{\lambda r}\right)\right)$ coincides with

$$
\left\{\left(x_{1}, x_{3}\right): \frac{\left|x_{1}\right|}{\lambda} \leq 1, \frac{\left|x_{3}\right|}{\lambda^{2}} \leq 1, \frac{\left|\phi^{2}\left(r x_{1}, r^{2} x_{3}\right)\right|}{\lambda r} \leq 1, \frac{\left|\phi^{4}\left(r x_{1}, r^{2} x_{3}\right)\right|}{(\lambda r)^{3}} \leq 1\right\}
$$


that contains the subset

$$
\left\{\left(x_{1}, x_{3}\right):\left|x_{1}\right| \leq \lambda,\left|x_{3}\right| \leq \lambda^{2},\left|\left(x_{1}, r x_{3}\right)\right| \leq \frac{\lambda}{c},\left|\left(x_{1}, r x_{3}\right)\right| \leq \sqrt{\frac{\lambda^{3} r}{c}}\right\} .
$$

We will denote this set by $S_{r}$. In view of the change of variable $x_{3}^{\prime}=r x_{3}$, it follows that the $\mathcal{L}^{2}$-measure of the set

$$
\left\{\left(x_{1}, x_{3}^{\prime}\right):\left|x_{1}\right| \leq \lambda,\left|x_{3}^{\prime}\right| \leq r \lambda^{2},\left|\left(x_{1}, x_{3}^{\prime}\right)\right| \leq \frac{\lambda}{c},\left|\left(x_{1}, x_{3}^{\prime}\right)\right| \leq \sqrt{\frac{\lambda^{3} r}{c}}\right\}
$$

is equal to $r \mathcal{L}^{2}\left(S_{r}\right)$. For $r>0$ small, it follows that

$$
\begin{aligned}
\mathcal{L}^{2}\left(S_{r}\right) & \geq \frac{1}{r} \mathcal{L}^{2}\left(\left\{\left(x_{1}, x_{3}\right):\left|x_{3}\right| \leq r \lambda^{2}, \max \left\{\left|x_{1}\right|,\left|x_{3}\right|\right\} \leq \sqrt{\frac{\lambda^{3} r}{2 c}}\right\}\right) \\
& =\frac{1}{r} \mathcal{L}^{2}\left(\left\{\left(x_{1}, x_{3}\right):\left|x_{3}\right| \leq r \lambda^{2},\left|x_{1}\right| \leq \sqrt{\frac{\lambda^{3} r}{2 c}}\right\}\right) \\
& =\frac{2^{3 / 2} \lambda^{7 / 2}}{\sqrt{c}} \sqrt{r}
\end{aligned}
$$

Finally, taking into account (4.7), (4.4) and (4.5), it follows that

$$
\frac{\mu_{2}\left(\Sigma \cap D_{x, r}\right)}{r^{d(\Sigma)}} \geq \frac{\mathcal{L}^{2}\left(S_{r}\right)}{r^{d(\Sigma)-3}} \frac{J \phi(0)}{2} \longrightarrow+\infty \quad \text { as } \quad r \rightarrow 0 .
$$

Proposition 9. Let $\Sigma$ be a 2-dimensional $C^{1,1}$ smooth submanifold of $\mathbb{E}$ and assume that $d(\Sigma)=3$ and $d_{\Sigma}(x)=2$. Then we have

$$
\lim _{r \rightarrow 0} \frac{\mu_{2}\left(\Sigma \cap D_{x, r}\right)}{r^{3}}=+\infty
$$

Proof . From Lemma 6, applying (4.2) and (4.3), we get

$$
\phi(u)=\left(u_{1}, u_{2}, \phi^{3}(u), \phi^{4}(u)\right)
$$

that parametrizes a neighbourhood of 0 in $x^{-1} \Sigma$ and there exists a constant $c>0$ such that $\left|\phi^{3}(u)\right| \leq c|u|^{2}$ and $\left|\phi^{4}(u)\right| \leq c|u|^{2}$ for $u$ small. Notice that these estimates do not suffice to obtain our claim. We have to exploit the assumption that $\Sigma$ has degree three. In fact, from (3.1) it follows that

$$
\left\{\begin{array}{l}
\phi_{u}^{14}-\phi^{1} \phi_{u}^{13}+\frac{\left(\phi^{1}\right)^{2}}{2} \phi_{u}^{12}=0 \\
\phi_{u}^{24}-\phi^{1} \phi_{u}^{23}=0 \\
\phi_{u}^{34}+\frac{\left(\phi^{1}\right)^{2}}{2} \phi_{u}^{23}-\phi^{1} \phi_{u}^{24}=0
\end{array} .\right.
$$


Thus, these equations hold for (4.8) and in particular the first two ones yield

$$
\left\{\begin{array}{l}
\phi_{u_{2}}^{4}-u_{1} \phi_{u_{2}}^{3}+\frac{\left(u_{1}\right)^{2}}{2}=0 \\
-\phi_{u_{1}}^{4}+u_{1} \phi_{u_{1}}^{3}=0
\end{array} .\right.
$$

We have proved that

$$
\nabla \phi^{4}=\left(u_{1} \phi_{u_{1}}^{3}, u_{1} \phi_{u_{2}}^{3}-\frac{\left(u_{1}\right)^{2}}{2}\right),
$$

hence the $C^{1,1}$ regularity of $\varphi$ yields a constant $c_{1}>0$ such that $\left|\phi^{4}(u)\right| \leq$ $c_{1}|u|^{3}$ for $u$ small. Now, we argue as in Proposition 7 , taking into account this better estimate on the order of vanishing of $\phi^{4}$. In this case, we have

$$
\begin{aligned}
& \tilde{\delta}_{\frac{1}{r}}\left(\phi^{-1}\left(\operatorname{Box}_{\lambda r}\right)\right)= \\
& =\left\{\left(x_{1}, x_{2}\right): \frac{\left|x_{1}\right|}{\lambda} \leq 1, \frac{\left|x_{2}\right|}{\lambda} \leq 1, \frac{\left|\phi^{3}\left(r x_{1}, r x_{2}\right)\right|}{(\lambda r)^{2}} \leq 1, \frac{\left|\phi^{4}\left(r x_{1}, r x_{2}\right)\right|}{(\lambda r)^{3}} \leq 1\right\} \\
& \supset\left\{\left(x_{1}, x_{2}\right):\left|x_{1}\right| \leq \lambda,\left|x_{2}\right| \leq \lambda,|x| \leq \frac{\lambda}{\sqrt{c}},|x| \leq \frac{\lambda}{\sqrt[3]{c_{1}}}\right\}=S,
\end{aligned}
$$

Then taking into account (4.4) and (4.5), it follows that

$$
\frac{\mu_{2}\left(\Sigma \cap D_{x, r}\right)}{r^{3}} \geq \frac{\mathcal{L}^{2}(S)}{r} \frac{J \phi(0)}{2} \longrightarrow+\infty \quad \text { as } \quad r \rightarrow 0 .
$$

Proof of Theorem 5. Notice that 2-dimensional $C^{1,1}$ submanifolds of degree 2 would be tangent everywhere to the horizontal subbundle, hence they cannot exist. Thus, the possible degrees of $\Sigma$ are 5,4 and 3 . If $d(\Sigma)=5$, then Theorem 2.16 of [4] applies, since the Hausdorff dimension $Q$ of $\mathbb{E}$ is 7, the codimension of $\Sigma$ is 2 and the set $C(\Sigma)$ in [4] coincides with $\Sigma \backslash \Sigma_{5}$. Then we have $\mathcal{S}^{5}\left(\Sigma \backslash \Sigma_{5}\right)=0$. If $d(\Sigma)=4$, then Propositions 7 and 8 imply that

$$
\lim _{r \rightarrow 0} \frac{\mu_{2}\left(\Sigma \cap D_{x, r}\right)}{r^{4}}=+\infty
$$

whenever $x \in\left\{z \in \Sigma \mid d_{\Sigma}(z)<4\right\}=\Sigma \backslash \Sigma_{4}$. Applying Lemma 4, we get $\mathcal{S}^{4}\left(\Sigma \backslash \Sigma_{4}\right)=0$. If $d(\Sigma)=3$, then Propositions 9 implies that

$$
\lim _{r \rightarrow 0} \frac{\mu_{2}\left(\Sigma \cap D_{x, r}\right)}{r^{3}}=+\infty
$$

whenever $x \in\left\{z \in \Sigma \mid d_{\Sigma}(z)<3\right\}=\Sigma \backslash \Sigma_{3}$. Then Lemma 4 yields $\mathcal{S}^{3}\left(\Sigma \backslash \Sigma_{3}\right)=0$. This ends the proof. 
Remark 2. Our techniques more precisely allow for extending both Proposition 7 and Proposition 8 to the case when $\Sigma$ is of class $C^{1, \alpha}$ and $\alpha>$ $(6-d(\Sigma)) / d(\Sigma)$. In the same way, Proposition 9 still holds for $C^{1, \alpha}$ surfaces with $\alpha>1 / 3$. We limit ourselves to surfaces of class $C^{1,1}$, since this regularity is needed to apply results of [5] and therefore to obtain formula (1.2) of Corollary 2.

\section{Curves in the Engel group}

In this section we wish to show the following

Theorem 10. Let $\Sigma$ be a 1-dimensional $C^{1,1}$ smooth submanifold of $\mathbb{E}$. Let $d$ be the degree of $\Sigma$ and let $\Sigma_{d}$ be the open subset of points of degree $d$. Then we have

$$
\mathcal{S}^{d}\left(\Sigma \backslash \Sigma_{d}\right)=0 .
$$

In analogy with comments of Section 4 , we will number the independent variable of curves taking into account the coordinate line it spans in $\mathbb{R}^{4}$. In fact, we consider curves locally presented as graphs embedded in $\mathbb{R}^{4}$. The indexes will also indicate the degree of that direction with respect to the Engel dilations of $\mathbb{R}^{4}$.

Lemma 11. Let $\Sigma$ be a 1-dimensional $C^{1}$ smooth submanifold of $\mathbb{E}$ and let $x \in \Sigma$. Then there exists a neighbourhood I of 0 in $\mathbb{R}$ such that $x^{-1} \Sigma$ around zero is given by the local parametrization $\phi: I \longrightarrow x^{-1} \Sigma$ with $\phi(0)=0$ and we have

$$
\phi= \begin{cases}\left(\phi^{1}\left(t_{4}\right), \phi^{2}\left(t_{4}\right), \phi^{3}\left(t_{4}\right), t_{4}\right) & \text { if } d_{\Sigma}(x)=3, \\ \left(\phi^{1}\left(t_{3}\right), \phi^{2}\left(t_{3}\right), t_{3}, \phi^{4}\left(t_{3}\right)\right) & \text { if } d_{\Sigma}(x)=2, \\ \left.\left(t_{1}, \phi^{2}\left(t_{1}\right), \phi^{3}\left(t_{1}\right), \phi^{4}\left(t_{1}\right)\right) \text { or }\left(\phi^{1}\left(t_{2}\right), t_{2}, \phi^{3}\left(t_{2}\right), \phi^{4}\left(t_{2}\right)\right)\right) & \text { if } d_{\Sigma}(x)=1,\end{cases}
$$

where the functions $\phi^{j}$ satisfy

$$
\left\{\begin{array}{ll}
\dot{\phi}^{4}(0)=0 & \text { if } d_{\Sigma}(x)=2 \\
\dot{\phi}^{4}(0)=\dot{\phi}^{3}(0)=0 & \text { if } d_{\Sigma}(x)=1
\end{array} .\right.
$$

Furthermore, for small $r>0$, we have

$$
\mu_{1}\left(D_{x, r} \cap \Sigma\right)=r^{d_{\Sigma}(x)} \int_{\tilde{\delta}_{1 / r}\left(\phi^{-1}\left(D_{r}\right)\right)} J \phi\left(\tilde{\delta}_{r} t\right) d t
$$

where $J \phi(t)=\sqrt{\langle\dot{\phi}(t), \dot{\phi}(t)\rangle}$ and $\langle$,$\rangle denotes the fixed left invariant Rie-$ mannian metric. The induced dilation is $\tilde{\delta}_{r}\left(t_{j}\right)=r^{d_{j}} t_{j}$, where $d_{1}=d_{2}=1$, $d_{3}=2$ and $d_{4}=3$. 
Proof. Taking into account the notion of pointwise degree, the proof of (5.2) simply follows from the implicit function theorem and formula (3.4). As in the proof of Lemma 6, we consider just one case as an example. The other ones can be simply obtained by repeating this argument. For instance, if $d_{\Sigma}(x)=3$, then $\dot{\psi}_{4}(0) \neq 0$, where $\psi$ is a parametrization of $x^{-1} \Sigma$ around the origin. Setting $t_{4}=\psi(s)$, we have proved (5.2) in the case $d_{\Sigma}(x)=3$. Now, if we apply (3.4) to the parametrization $\phi$ at the origin, then a simple computation gives (5.3). In fact, one has applied (3.4) to all forms of $\phi$ listed in (5.2). The left invariance of the Riemannian surface measure gives

$$
\mu_{1}\left(\Sigma \cap D_{x, r}\right)=\mu_{1}\left(x^{-1} \Sigma \cap D_{r}\right)=\int_{\phi^{-1}\left(D_{r}\right)} J \phi(t) d t .
$$

The change of variable $\tilde{t}_{j}=r^{d_{j}} t_{j}$ and the fact that $d_{\Sigma}(x)=d_{j}$ lead us to formula (5.4).

Proposition 12. Let $\Sigma$ be a 1-dimensional $C^{1,1}$ smooth submanifold of $\mathbb{E}$ and assume that $d(\Sigma) \geq 2$ and $d_{\Sigma}(x)=1$. Then we have

$$
\lim _{r \rightarrow 0} \frac{\mu_{1}\left(\Sigma \cap D_{x, r}\right)}{r^{d(\Sigma)}}=+\infty
$$

Proof. By Lemma 11, applying formulae (5.2) and (5.3), we get a constant $c>0$ such that $\max \left\{\left|\phi^{3}(t)\right|,\left|\phi^{4}(t)\right|\right\} \leq c|t|^{2}$ and $\left|\phi^{2}(t)\right| \leq c|t|$ for $t$ small. Moreover, $x^{-1} \Sigma$ can be locally parametrized either by

$$
\left(t_{1}, \phi^{2}\left(t_{1}\right), \phi^{3}\left(t_{1}\right), \phi^{4}\left(t_{1}\right)\right) \quad \text { or } \quad\left(\phi^{1}\left(t_{2}\right), t_{2}, \phi^{3}\left(t_{2}\right), \phi^{4}\left(t_{2}\right)\right) .
$$

Clearly, both cases have the same proof, hence assume for instance that

$$
\phi\left(t_{1}\right)=\left(t_{1}, \phi^{2}\left(t_{1}\right), \phi^{3}\left(t_{1}\right), \phi^{4}\left(t_{1}\right)\right)
$$

locally parametrizes $x^{-1} \Sigma$. Taking into account (4.5), we consider

$$
\tilde{\delta}_{\frac{1}{r}}\left(\phi^{-1}\left(\operatorname{Box}_{\lambda r}\right)\right)=\tilde{\delta}_{1 / r}\left\{t_{1}: \frac{\left|t_{1}\right|}{\lambda r} \leq 1, \frac{\left|\phi^{2}\left(t_{1}\right)\right|}{\lambda r} \leq 1, \frac{\left|\phi^{3}\left(t_{1}\right)\right|}{(\lambda r)^{2}} \leq 1, \frac{\left|\phi^{4}\left(t_{1}\right)\right|}{(\lambda r)^{3}} \leq 1\right\} .
$$

This set can be written as

$$
\left\{\tau: \frac{|\tau|}{\lambda} \leq 1, \frac{\left|\phi^{2}(r \tau)\right|}{\lambda r} \leq 1, \frac{\left|\phi^{3}(r \tau)\right|}{(\lambda r)^{2}} \leq 1, \frac{\left|\phi^{4}(r \tau)\right|}{(\lambda r)^{3}} \leq 1\right\} .
$$

For $r>0$ small, the previous set contains

$$
S_{r}=\left\{\tau:|\tau| \leq \frac{\lambda^{3 / 2}}{\sqrt{c}} \sqrt{r}\right\}
$$


Taking into account (5.4) and (4.5), it follows that

$$
\frac{\mu_{1}\left(\Sigma \cap D_{x, r}\right)}{r^{d(\Sigma)}} \geq \frac{\mathcal{L}^{1}\left(S_{r}\right)}{r^{d(\Sigma)-1}} \frac{J \phi(0)}{2} \longrightarrow+\infty \quad \text { as } \quad r \rightarrow 0 .
$$

Proposition 13. Let $\Sigma$ be a 1-dimensional $C^{1,1}$ smooth submanifold of $\mathbb{E}$ and assume that $d(\Sigma)=3$ and $d_{\Sigma}(x)=2$. Then we have

$$
\lim _{r \rightarrow 0} \frac{\mu_{1}\left(\Sigma \cap D_{x, r}\right)}{r^{3}}=+\infty \text {. }
$$

Proof. By Lemma 11, applying formulae (5.2) and (5.3), we get a constant $c>0$ such that $\left|\phi^{4}(t)\right| \leq c|t|^{2}$ and $\max \left\{\left|\phi^{1}(t)\right|,\left|\phi^{2}(t)\right|\right\} \leq c|t|$ for $t$ small. Moreover, $x^{-1} \Sigma$ can be locally parametrized by

$$
\left(\phi^{1}\left(t_{3}\right), \phi^{2}\left(t_{3}\right), t_{3}, \phi^{4}\left(t_{3}\right)\right)
$$

Taking into account the previous estimates on $\phi^{j}$, for $r>0$ small we have

$$
\begin{aligned}
& \tilde{\delta}_{\frac{1}{r}}\left(\phi^{-1}\left(\operatorname{Box}_{\lambda r}\right)\right)= \\
& \quad=\tilde{\delta}_{1 / r}\left\{t_{3}: \frac{\left|\phi^{1}\left(t_{3}\right)\right|}{\lambda r} \leq 1, \frac{\left|\phi^{2}\left(t_{3}\right)\right|}{\lambda r} \leq 1, \frac{\left|t_{3}\right|}{(\lambda r)^{2}} \leq 1, \frac{\left|\phi^{4}\left(t_{3}\right)\right|}{(\lambda r)^{3}} \leq 1\right\} \\
& \quad=\left\{\tau:|\tau| \leq \lambda^{2}\right\}=S .
\end{aligned}
$$

Thus, from (5.4) and (4.5), it follows that

$$
\frac{\mu_{1}\left(\Sigma \cap D_{x, r}\right)}{r^{3}} \geq \frac{\mathcal{L}^{1}(S)}{r} \frac{J \phi(0)}{2} \longrightarrow+\infty \quad \text { as } \quad r \rightarrow 0 .
$$

Proof of Theorem 10. If $d(\Sigma)=3$, then Propositions 12 and 9 imply that

$$
\lim _{r \rightarrow 0} \frac{\mu_{1}\left(\Sigma \cap D_{x, r}\right)}{r^{3}}=+\infty,
$$

whenever $x \in\left\{z \in \Sigma \mid d_{\Sigma}(z)<4\right\}=\Sigma \backslash \Sigma_{4}$. Thus, Lemma 4 yields $\mathcal{S}^{3}\left(\Sigma \backslash \Sigma_{3}\right)=0$. If $d(\Sigma)=2$, then Proposition 12 implies that

$$
\lim _{r \rightarrow 0} \frac{\mu_{2}\left(\Sigma \cap D_{x, r}\right)}{r^{2}}=+\infty,
$$

whenever $x \in\left\{z \in \Sigma \mid d_{\Sigma}(z)<2\right\}=\Sigma \backslash \Sigma_{2}$ and this gives $\mathcal{S}^{2}\left(\Sigma \backslash \Sigma_{2}\right)=0$.

Remark 3. As in the previous section, we observe that our techniques more precisely allow for extending Proposition 12 to the case when the curve $\Sigma$ is of class $C^{1, \alpha}$ and $\alpha>(3-d(\Sigma)) / d(\Sigma)$. In the same way, Proposition 13 still holds for $C^{1}$ curves. Also in this case, we have fixed our attention on $C^{1,1}$ curves to apply results of [5] that lead us to the integral formula (1.2) for curves.

Acknowledgements. The authors thank the referees for their interesting comments. 


\section{References}

[1] Balogh, Z. M., Tyson, J. T. and Warhurst, B.: Sub-Riemannian vs Euclidean dimension comparison and fractal geometry on Carnot groups. Adv. Math. 220 (2009), no. 2, 560-619.

[2] Federer, H.: Geometric measure theory. Die Grundlehren der mathematischen Wissenschaften 153. Springer-Verlag, New York, 1969.

[3] Gromov, M.: Carnot-Carathéodory spaces seen from within. In SubRiemannian geometry, 79-323. Progress in Mathematics 144. Birkhauser, Basel, 1996.

[4] Magnani, V.: Characteristic points, rectifiability and perimeter measure on stratified groups. J. Eur. Math. Soc. 8 (2006), no. 4, 585-609.

[5] Magnani, V. And Vittone, D.: An intrinsic measure for submanifolds in stratified groups. J. Reine Angew. Math. 619 (2008), 203-232.

[6] Magnani, V.: Non-horizontal submanifolds and coarea formula. J. Anal. Math. 106 (2008), 95-127.

[7] Magnani, V.: Blow-up estimates at horizontal points and applications. To appear in J. Geom Anal.

Recibido: 11 de septiembre de 2008

Revisado: 17 de noviembre de 2008

Enrico Le Donne Department of Mathematics

ETH, Zürich

HG G 68.1

Rämistrasse 101

8092 Zürich, Switzerland enrico.ledonne@math.ethz.ch

Valentino Magnani Dipartimento di Matematica

Università di Pisa

Largo Pontecorvo 5

I-56127, Pisa

magnani@dm . unipi.it 